\title{
Motion of Grinding Media in Axial Direction and its Effect on Comminution in an Agitation Bead Mill ${ }^{\dagger}$
}

\author{
Yoko Hashi and Mamoru Senna
}

Faculty of Science and Technology, Keio University*

\begin{abstract}
Grinding tests were carried out using a model agitation bead mill with a single disc. Four different types of disc were used to examine the effect of the motion of the grinding media in the axial direction. The median diameter of the ground product decreased with increasing axial movement of the grinding media under the same degree of filling of the grinding media and rate of rotation. Contamination in the product from the agitating element and grinding vessel increased with increasing energy input, irrespective of the shape of the agitating disc. Contamination from the grinding media increased with an increase in the axial movement of the grinding media which promoted mutual collision and hence abrasion of the grinding media.
\end{abstract}

\section{Introduction}

By observing the actual performance in agitation vessels made of transparent material, it turned out that agitation bead mills use shear friction between grinding media and/or vertical compressive force during grinding ${ }^{3-7}$ ). Motion of the grinding media in an agitation bead mill has been discussed based on the agitation theory ${ }^{1,2)}$. The predominating motion of the grinding media in an agitation vessel is circular, while the radial motion is subordinate. The latter is attributed to disc rotation, circulation flow in the axial or vertical direction, and local and irregular motions due to the presence of pin-like structures. Because of these complex motions of the grinding media, it has not been clearly demonstrated what kind of motion predominated in the comminution effect.

There is a view that the particle diameter of the ground product is determined by the input energy, and not by the type of mill or operating conditions ${ }^{8)}$. However, this is not very applicable to local regions where the motions of the grinding media are different from each other.

Tenda et al. ${ }^{7)}$ measured the circular component of the speed of the grinding media, in a region where grinding operation was highly effective in a vertical type agitation bead mill. They revealed from the experiments using a vessel of $2.2 \mathrm{dm}^{3}$ and three different discs-horizontal plate, vertical pin and horizontal pin - that grinding efficiency depends on

* 3-14-1 Hiyoshi, Kohoku-ku, Yokohama 223 JAPAN

$\uparrow$ This report was originally printed in Kagaku Kougaku Ronbunshu, 21, 502 (1995) in Japanese, before being translated into English by KONA Editorial Committee with the permission of the editorial committee of the Soc. Chemical Engineers, Japan. the fluctuation amplitude of the grinding media rather than the average velocity or velocity gradient.

We devised a single disc type agitation bead mill that enables simple motion of grinding media and allows independent regulation of radial and axial velocities. Using the model mill, we measured the energy consumption in the grinding vessel, particle size of the ground product and contamination from the grinding media. The main circular motion combined with several subordinate motions of the grinding media was simulated as well.

\section{Experimental equipment and method}

The model mill has a grinding chamber shown in Figure 1. All parts contacting with the slurry of the raw material are made of SUS304. The disc is

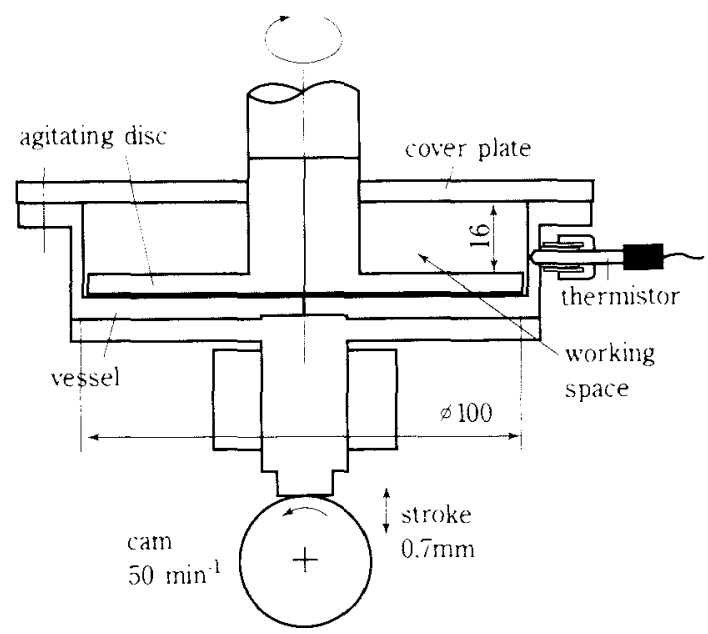

Fig. 1 Scheme of single disc mill 
Connected to the lower end of the driving shaft. The vessel is connected by a rod with a cam which enables a periodical vertical motion. The clearance between the wall of the vessel and the disc edge is less than one-third of the particle diameter of the grinding media so that particles are not captured under the disc. The volume of the grinding vessel refers to the space between the upper plate of the vessel and disc top. The cylindrical part of the vessel has an opening for a temperature sensor and is insulated to avoid heat transfer through the wall. Table 1 shows the main dimensions of the vessel, operating conditions and the sample specification. Figure $\mathbf{2}$ shows the discs we used. The experiment with vessel vertical motion was performed only with the plain disc.

The sample to be ground was a mixture of water and calcium carbonate (NS100 by Nitto Funka) at a solid volume fraction 0.1 . The initial size distribution of the calcium carbonate was $0.98 \mu \mathrm{m}, 5.12 \mu \mathrm{m}$ and $14.5 \mu \mathrm{m}$ in $10 \%, 50 \%$ and $90 \%$ cumulative values, respectively. Alumina beads with their diameter ranging from $1.7 \mathrm{~mm}$ to $2.0 \mathrm{~mm}$ (D2 by Ashizawa Ltd.) were used as the grinding media. They contained about $13 \%$ silica. The vessel was filled with the slurry after feeding the required amount of the grinding media. Operation time was 15 minutes. The temperature in the vessel and particle size of the sample were monitored during the operation. A laser diffraction system (Micro-Trac Spa by Leeds \& Northrup Instruments) was used for particle size analysis. The concentration of $F e$ and $A l$ was measured using a fluorescent X-ray analyzing system (3080E2 by Rigaku Electric Equipment Ltd.).

\section{Motion of grinding medium}

According to our previous papers ${ }^{5,7)}$, the tangential component of the motion of the grinding media is more than twice as large as that in either the axial (vertical) or radial direction. In the case with the flat disc and the vessel agitated without vertical movement, axial and radial components are even smaller, as shown in Figure. 3(a). The motion of the grinding media are, therefore, nearly tangential.

In the case of agitating the vessel with the flat disc moving periodically in a vertical direction, the entire grinding media moved in the axial direction periodically, compressing and decompressing throughout the vessel, as shown in Figure. 3(b). The compression frequency was the same with that of the vertical motion.

Table 1 Apparatus dimensions and operating conditions

Vessel diameter

Vessel length

Working volume

Rotating speed

Vessel axial slide frequency stroke

Grinding media

Weight of grinding media

Weight of water

Weight of powder
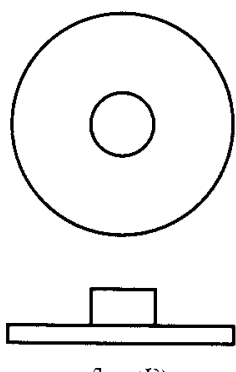

flat $(F)$
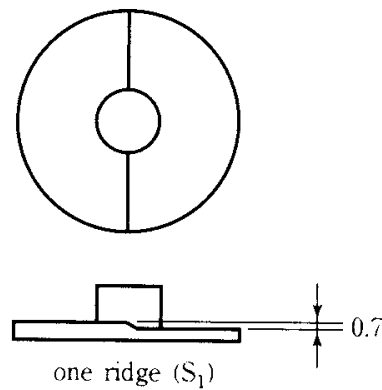

flat disc with $0.7 \mathrm{~mm}$ stroke (M)

Fig. 2 Agitating discs

In the case of the inclined disc without vertical motion of the vessel, grinding media were compressed and decompressed periodically. This differed from the previous case with simultaneous compression and decompression, and, depending on the position of the disc, force acted in the tangential and axial directions. The compression/decompression frequency of the grinding media was larger than the rotating frequency of the disc because of the circular motion of the grinding media. The compression/decompression frequency was nearly one-tenth of that of the vessel vertical motion.

With the ridged discs, the direction of the motion of some particles changes suddenly as shown in 

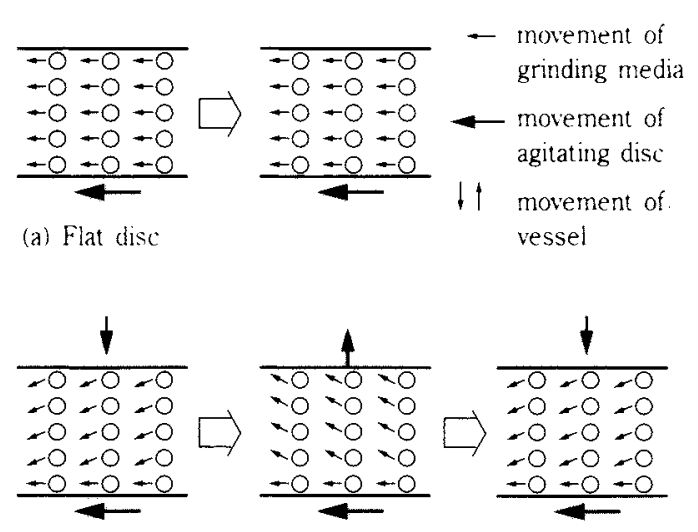

(b) Flat disc with slide of vessel

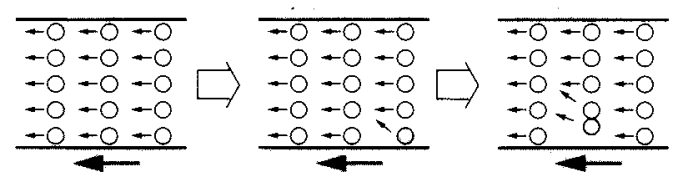

(c) Ridged disc

Fig. 3 Movement of grinding media

Figure 3(c). The particles then collide with each other, which disturbs media configuration. A disc having six ridges caused an axial motion six times more frequent.

\section{Results and discussions}

\subsection{Temperature of the sample during the experiment}

Since the energy input to the grinding vessel was too small to be evaluated with reasonable accuracy, we measured the temperature rise as an indirect measure of energy consumption. Figure 4(a) shows the case with the smallest change in temperature, while the Figure 4(b) the largest. The other two cases are in between. The temperature rise due to the type of disc is the largest for S6 (six ridges), followed by $\mathrm{S} 1$ (single ridge), $T$ (inclined), $M$ (flat and vertical motion) and $F$ (flat). The rate of temperature rise decreases at higher temperatures as shown in Figure 4(b). This is attributed to energy dissipation by poor heat insulation and thermal conduction through the drive shaft. For the cases with smaller temperature rise, the heat dissipation is small.

The ratio of the heat dissipation to the total heat generation in the vessel per unit time is less than 0.05 for a temperature difference of $5 \mathrm{~K}$. This means that temperature rise is proportional to heat genera-

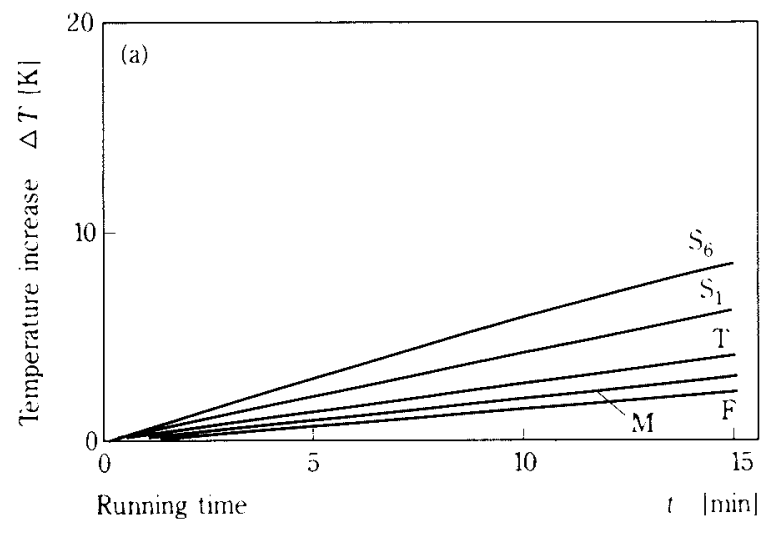

(a) $\Phi_{n}=0.4, n=1350 \mathrm{~min}^{-1}$

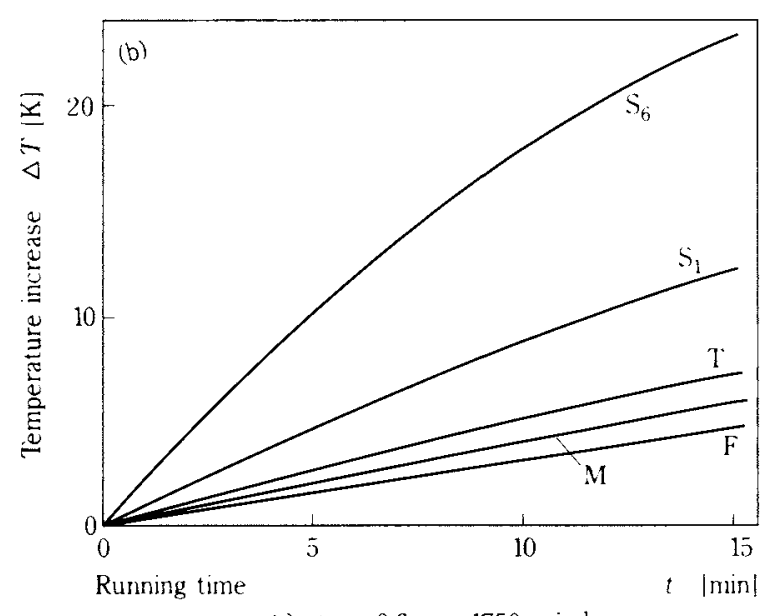

(b) $\phi_{n}=0.6, n=1750 \mathrm{~min}^{-1}$

Fig. 4 Temperature increase of slurry

tion within a $5 \%$ tolerance.

Most of the energy consumed in the vessel is transformed into heat, while only a small amount of energy is used for grinding. Therefore, the energy consumption is considered proportional to temperature rise within the same $5 \%$ tolerance.

\subsection{Particle size distribution after grinding}

Figure 5(a) shows the particle size distribution with the smallest load among the four cases. Figure 5(a) shows no significant difference among those obtained when using $\operatorname{discs} F, M$ and $T$. The particle size distribution is not affected by rotating speed $\left(1350-1750 \mathrm{~min}^{-1}\right)$ or filling rate $(0.4-0.6)$. Since energy consumption depends on the type of disc as shown in 4.1 , we concluded that the increase in energy consumption does not contribute to the grinding operation. In Figure 5(b), no significant 

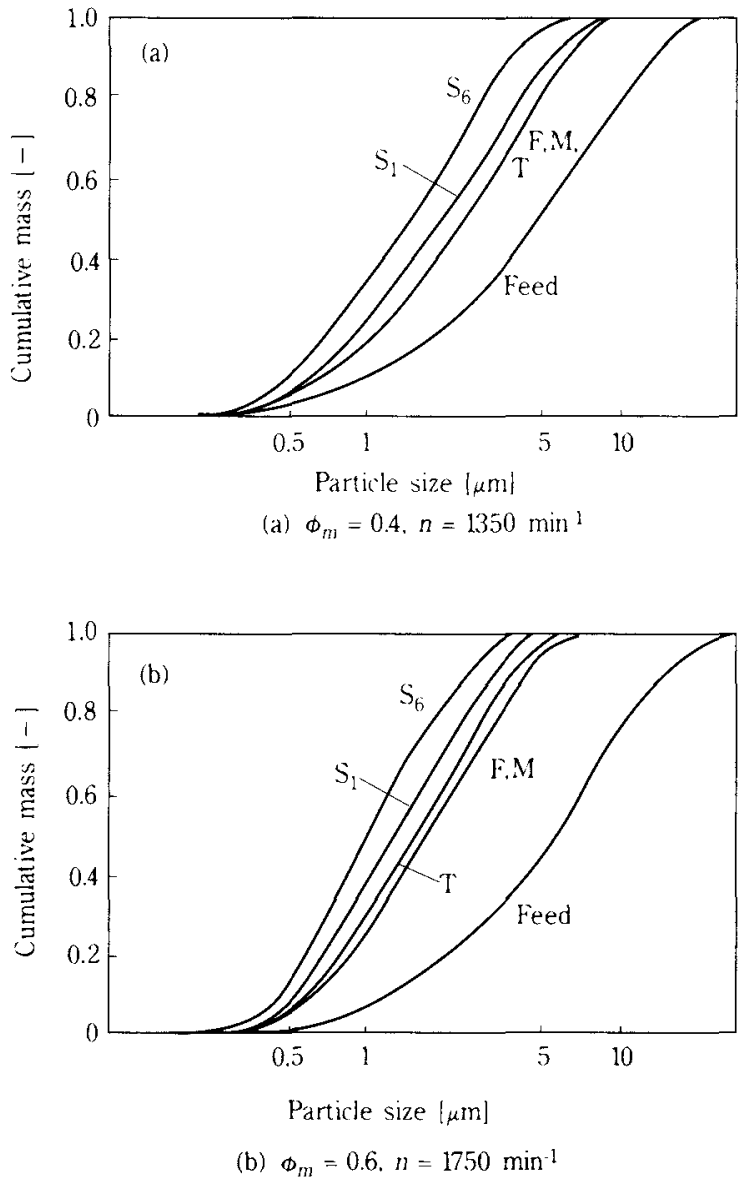

Fig. 5 Particle size distribution of prodcut

difference in the particle distribution is found between $\operatorname{discs} F$ and $M$, while disc $T$ causes a different distribution from others. This means that the inclined disc $T$ improves grinding efficiency at a higher filling rates and rotating speed.

Ridged discs result in smaller particles, being capable of abrupt change in the motion of the grinding media and improving the grinding effect significantly.

Figure 6 shows the relationship between particle size and slurry temperature measured five minutes after the start of operation. The larger temperature rise render and a higher filling rate a smaller particle size.

\subsection{Sample contamination}

Figure 7 shows the relationship between iron $\mathrm{Fe}$ concentration and energy input. The $\mathrm{Fe}$ and $\mathrm{Al}$ concentrations are normalized with respect to the mass of the slurry. Temperature rise $\left(\Delta T_{5}\right)$ up to $2 \mathrm{~K}$ rarely causes a change in $\mathrm{Fe}$ concentration. Above $2 K, \Delta T_{5}$ is proportional to $F e$ concentration. Thus, abrasion is negligible when energy consumption is

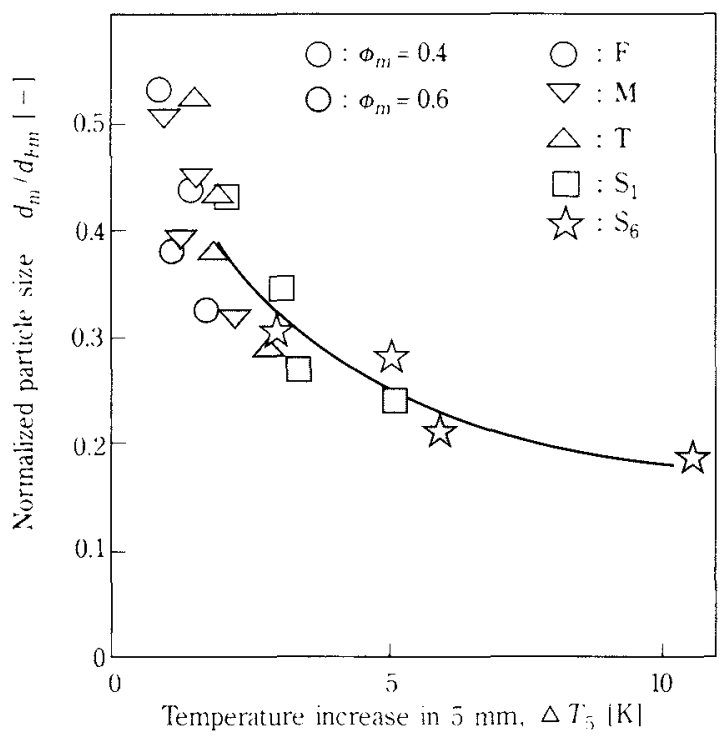

Fig. 6 Relationship between particle size and energy input

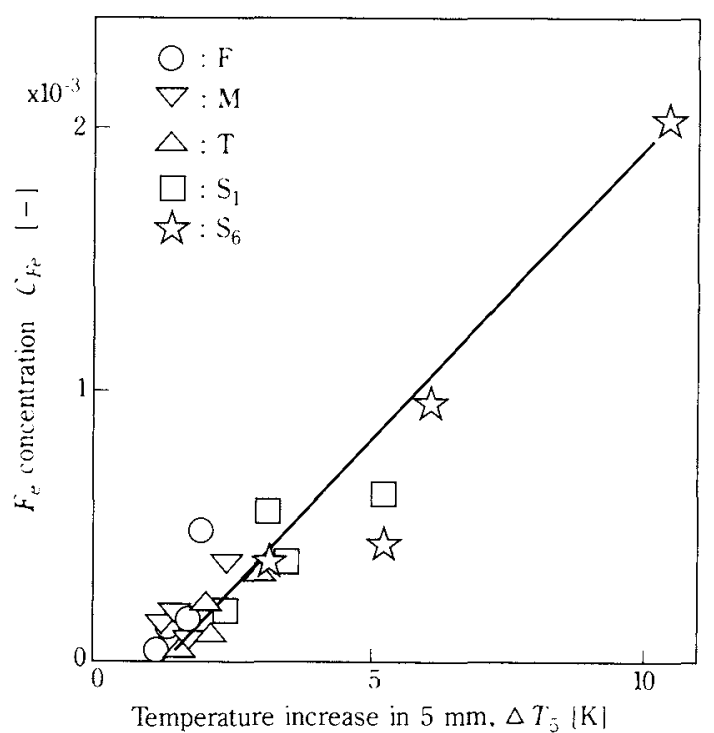

Fig. 7 Relationship between $F e$ concentration and energy input less than a certain value.

Figure 8 shows the relationship between $A l$ and $\mathrm{Fe}$ concentrations in the sample. Note that aluminum is contained only in the grinding medium. Al contamination is therefore attributed to the friction within the grinding media itself and/or between the media and the wall. Iron $(\mathrm{Fe})$ contamination is exclusively originated from the machine.

Both $F e$ and $A l$ concentrations increase concurrently 


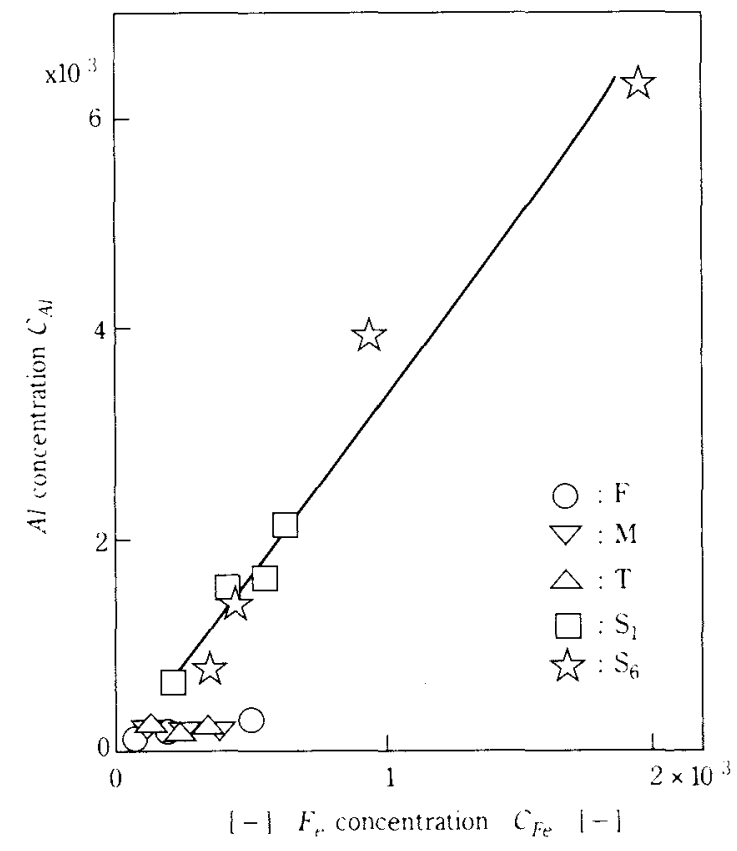

Fig. 8 Relationship between $A l$ and $F e$ concentration

when using $S 1$ or $S 6$ ridged discs, while only $F e$ concentration increases in the case of discs $F, M$ or $T$. This means that using an $S 1$ or $S 6$ disc causes more abrasion between the grinding media or between the media and the machine, as energy consumption increases. Moreover, $\operatorname{discs} F, M$ and $T$ rarely increase abrasion even when the energy consumption increases. The relationship between $\mathrm{Fe}$ and $\mathrm{Al}$ concentration proves that the ridged discs causing sudden changes in the media motion, trigger more frequent and intensive collisions between grinding media. From this result and the particle size distribution described in Section 4.2, we concluded that collisions of the grinding media due to the sudden axial motion promote the size reduction.

Figure 9 shows the relationship between the particle size and $A l$ concentration. It was shown in 4.2 that ridged discs are better than other types of disc in terms of grinding efficiency. However, even with the discs without ridges, the change in the filling rate and/or rotating speed can also improve grinding efficiency to the same level achieved by the ridged disc, judging from the initial/final particle size ratio, 0.3 (Figure 9). Figure 9 also shows that using a non-ridged disc reduces $A l$ concentration at the same initial/final particle size ratio. It is, therefore, recommended to use a non-ridged disc, whenever it suffices in order to avoid product contamination.

Figure 10 shows the relationship between the particle size and the total amount of impurity fraction,

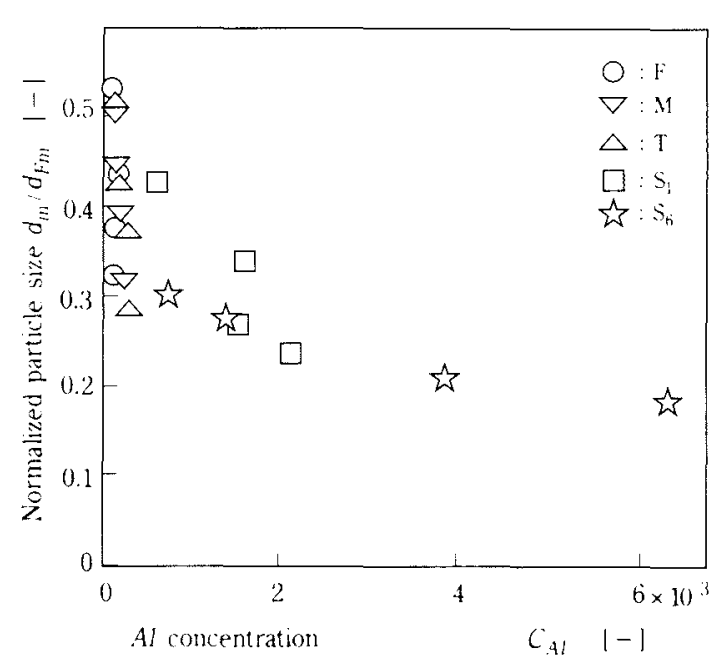

Fig. 9 Relationship between particle size and $A l$ concentration

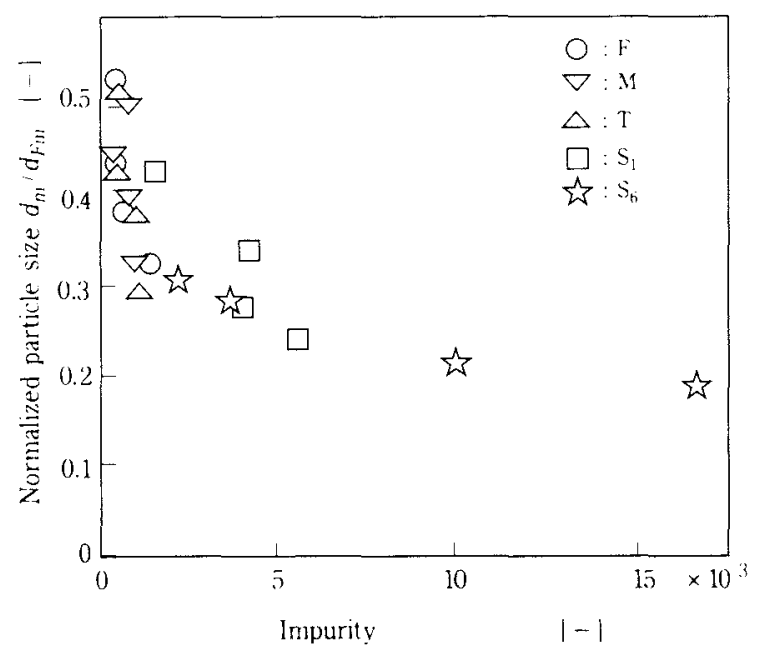

Fig. 10 Relationship between particle size and impurity of slurry

calculated from the $\mathrm{Al}$ and $\mathrm{Fe}$ concentration. Contamination from the grinding media is dominant as compared with those from the machine, as mentioned previously.

\section{Conclusion}

When powders are ground in an agitation bead mill at constant filling rate and constant rotating speed, a sudden vertical (axial) motion of the grinding media under circular motion promotes size reduction. Contamination. from the machine increases with the energy consumption for any type of disc. Contamination from the grinding media increases by using, a ridged disc. It is therefore recommended to use a non-ridged disc as far as the particle size range reached is satisfactory. 


\section{Nomenclature}

$C_{A l}=$ relative concentration of $A I$ in product $[-]$

$C_{F e}=$ relative concentration of $F e$ in product [-]

$d_{F m}=$ median particle size of feed $\quad[\mu \mathrm{m}]$

$d_{m}=$ median particle size of product $\quad[\mu \mathrm{m}]$

$n=$ speed of rotation

$t=$ running time

$\Delta T=$ temperature increase

$\Delta T_{5}=$ temperature increase in $5 \mathrm{~min}$

$\phi_{m}=$ filling degree of grinding media

\section{Literature cited}

1) Bosse, D.G.: Official Digest, 30, 250-276 (1958)

2) Engels, K.: Farbe u. Lack, 71, 375-385 (1965)

3) Gondoh, T., M. Toh and Y. Murakami: Shikizai Kyokaishi, 62, 202-207 (1989)

4) Gondoh, T., M. Toh, Y. Murakami, Y. Hashi and M. Kusunoki: ibid., 64, 297-304 (1991)

5) Hashi, Y. and M. Senna: J. Soc. Powder Technol., Japan, 28, 738-744 (1991)

6) Kaku, M., Y. Murakami, T. Tenda, T. Gondoh, M. Toh and M. Kusunoki: Shikizai Kyokaishi, 63, 3-10 (1990)

7) Tenda, T., Y. Kubozono, H. Nishimura, T. Teshiba, S. Komori and Y. Murakami: ibid., 64, 148-155 (1991)

8) Weit, H. and J. Schwedes: Chem.-Ing.-Tech., 58, 818-819 (1986)

\section{Author's short biography}

\section{Mamoru Senna, PhD}

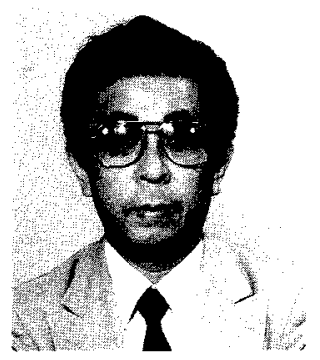

Graduated from Keio University, Graduate School in 1970, with a promotion to $\mathrm{PhD}$. He has been a visiting scientist from 1972 to 1974 in Technische Hochschule Aachen and from 1980 to 1981 in Technische Universität in Karlsruhe. Since 1988, he is a professor in the Department of Applied Chemistry, Faculty of Science and Technology, Keio Universisty. Enhancement of reactivity of solids by mechanical and irradiational strategy, including softmechanochemical synthesis center his research interests. 